\title{
Federating Distributed Clinical Data for the Prediction of Adverse Hypotensive Events
}

\author{
By Anthony Stell ${ }^{1}$, Richard Sinnott ${ }^{1}$, Jipu Jiang ${ }^{1}$, Rob Donald ${ }^{2,11}$, \\ Iain Chambers ${ }^{3}$, Giuseppe Citerio $^{4}$, Per Enblad ${ }^{5}$, Barbara Gregson ${ }^{6}$, \\ Tim Howells ${ }^{5}$, Karl Kiening ${ }^{7}$, Pelle Nilsson ${ }^{5}$, Arminas Ragauskas $^{8}$, \\ Juan Sahuquillo ${ }^{9}$, Ian Piper ${ }^{10}$ (on Behalf of the Brainit group) \\ ${ }^{1}$ National e-Science Centre, University of Glasgow, Glasgow, UK \\ ${ }^{2}$ C3 Amulet Ltd, Dingwall, UK \\ ${ }^{3}$ The James Cook University Hospital, Middlesbrough, UK \\ ${ }^{4}$ Neurorianimazione, Hospital San Gerardo, Monza, Italy \\ ${ }^{5}$ Neurosurgery, Uppsala University Hospital, Uppsala, Sweden \\ ${ }^{6}$ Neurosurgery, Newcastle General Hospital, Newcastle, UK \\ ${ }^{7}$ Neurosurgery, Ruprecht-Karls-Universitat Hospital Heidelberg, Germany \\ ${ }^{8}$ Kaunas University Hospital, Kaunas, Lithuania \\ ${ }^{9}$ Neurosurgery, Vall d'Hebron University Hospital, Barcelona, Spain \\ ${ }^{10}$ Clinical Physics, Southern General Hospital, Glasgow, UK \\ ${ }^{11}$ Dept of Statistics, University of Glasgow, Glasgow, UK
}

The ability to predict adverse hypotensive events, where a patient's arterial blood pressure drops to abnormally low (and dangerous) levels, would be of major benefit to the fields of primary and secondary health-care, and especially to the traumatic brain injury domain. A wealth of data exists in healthcare systems providing information on the major health indicators of patients in hospitals (blood pressure, temperature, heart-rate etc). It is believed that if enough of this data could be drawn together and analysed in a systematic way, then a system could be built that will trigger an alarm predicting the onset of a hypotensive event over a useful timescale, e.g. half an hour in advance. In such circumstances avoidance measures can be taken to prevent such events arising. This is the basis for the Avert-IT project (http://www.avert-it.org), a collaborative EU-funded project involving the construction of a hypotension alarm system exploiting Bayesian neural networks using techniques of data federation to bring together the relevant information for study and system development.

Keywords: Data Federation, Hypotension Prediction

\section{Introduction}

Prediction of detrimental changes to the health of the patient is of paramount importance in all clinical care. Hypotension, where a patient's blood pressure is abnormally low, is a condition that commonly occurs in intensive and high-dependency care units. Emergency treatments of hypotension are often highly invasive and carry 
associated risks of secondary complications including: tachycardia, peripheral vasoconstriction and cardiac ischaemia. To be able to predict the onset of a hypotensive event would be of clear benefit to a patient's outcome and this, in turn, has been estimated could translate to a reduction in ICU length of stay and thus a cost saving of an average of 1600 per patient per day across the EU25 [1].

To enable the prediction of these events through the use of a novel bed-side alarm system is the central premise of the Avert-IT project (Advanced Arterial Hypotension Adverse Event prediction through a Novel Bayesian Neural Network). There are a number of reactive bed-side systems currently in use, which typically sound an alarm as soon as a patient's vital signs indicate that they have entered a hypotensive phase. An example of such a system is the Odin monitor and browser [2]. In the intensive care management of patients with traumatic brain injury (TBI), the actual definition of a clinically significant hypotensive event can vary widely from centre to centre the partial standardisation of which across the participating clinical centres is itself a research outcome of the Avert-IT project (see $\S 2$ ). However, no system currently exists that can predict the onset of an adverse hypotensive event.

There are a variety of commercial systems available which allow the prediction of forthcoming clinical states. For example the BioSign device [3], which produces an index predicting cardiovascular instability based on several vital signs such as heart rate, respiration rate, etc. and the Philips Medical Event Surveillance Monitor [4], which supports the manual correlation of patient parameters into discrete "Events". However, none of these systems provide any probabilistic measure of the causative information of the events occurring and therefore cannot be tied to the context.

There are also a number of medical and clinical research groups that are attempting to enumerate the correlation of patient parameters such as blood pressure and heart rate to the onset of hypotension, but these are non-automated [5-6]. Therefore, a gap in the process of patient diagnosis has been identified: the automated prediction of hypotension in a patient. It is this gap that the Avert-IT project is attempting to address, by producing a predictive system, trained through an appropriate decision-support tool, on the data provided by six specialist centres from across Europe.

The next section of this paper outlines the clinical context and results obtained from research conducted into the definitions of hypotensive events. This in turn impacts the distribution of cases and data to be used later in the project. $\S 3$ details the computational requirements of the project, with $\S 4$ discussing the security issues in particular. $\S 5$ outlines the implementation of the proposed infrastructure and shows the progress to date.

\section{Clinical Brain Trauma Context}

In order to federate the necessary clinical data and use this to predict future events, the project consortium must first prioritise what that data should be. To do this, the definition of an event and identification of possible causal data must be agreed upon.

Article submitted to Royal Society 


\section{(a) Definition of a Hypotensive Event}

The generally accepted verbal definition of hypotension can be expressed as an abnormal drop in a patient's blood pressure. Although there are guidelines defining hypertension [7] and accepted ranges for what is considered normal blood pressure, there is no accepted standard definition for hypotension. In the management of patients with TBI, some guidelines suggest maintaining a patients systolic blood pressure above 90 [8] or even $120 \mathrm{mmHg}$ [9] but variability in following management guidelines for patients with TBI is considerable [10-11]. Thus there appears to be no general numerical consensus on what constitutes a hypotensive event in terms of the various measurements of blood pressure (systolic, diastolic or mean).

A survey conducted between the partners involved in Avert-IT showed a variation in using the systolic and mean blood pressures and cerebral perfusion pressure (CPP) values as indicators for a hypotensive event, shown in table 1, with the definition of the event terms used shown in figure 1.

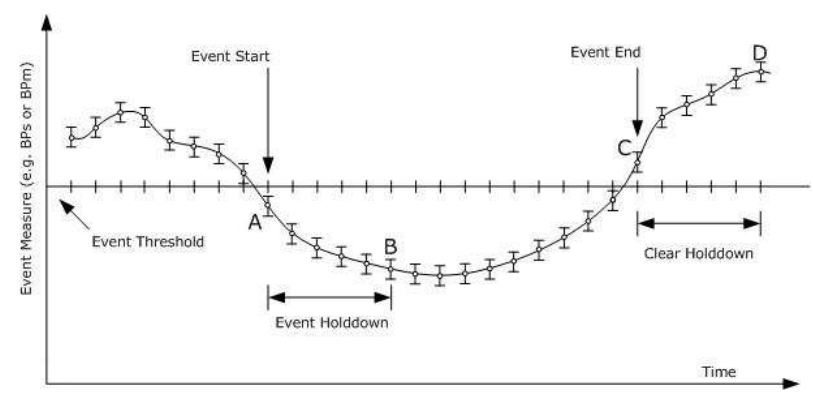

Figure 1. Hypotensive event definition

The Avert-IT project has been closely linked to the BrainIT project [12] - an internet based group set up to work collaboratively on standards for the collection and analyses of data from brain-injured patients towards providing a more efficient infrastructure for assessing new health technology [13]. As such, a study was conducted over the database created by this group to discover the spread of hypotensive events as defined by the different centre thresholds shown in table 1 . The spread of these results from the Avert-IT centres (within BrainIT) is shown in figure 2 .

Concern over the technical accuracy of measuring systolic blood pressure, led some clinicians to recommend including a blood pressure mean component to the definition. Such an approach was adopted by the group in Edinburgh and became standard as published in the Edinburgh University Secondary Insult Grading System (EUSIG) [15]. Therefore, further research was conducted upon the BrainIT database using four definitions of hypotensive events, based upon the EUSIG scoring system, which are shown in table 2.

From these results, and those expanded on in [14], the EUSIG definition of less than $90 \mathrm{mmHg}$ over 5 mins $(90 ; 5)$ for a hypotensive event threshold appears to be the most appropriate for training the decision-support tool later in the project. The fact that all four definitions produced similar numbers supports the decision to 
Table 1. Hypotensive event definitions

\begin{tabular}{|c|c|c|c|}
\hline Parameter & Uppsala & Glasgow & Kaunas \\
\hline Measure \& Threshold & BPs $<100$ & BPm $<70$ & $B P s / B P d<90 / 50$ \\
\hline Event hold down & 2 & 5 & 5 \\
\hline Clear hold down & BPs $>100 ; 5 \mathrm{~m}$ & BPm $>70 ; 5 \mathrm{~m}$ & BPm $>70 ; 5 \mathrm{~m}$ \\
\hline \hline Parameter & Heidelberg & Monza & Barcelona \\
\hline Measure \& Threshold & CPP $<50$ & BPs $<90$ & BPs $<90$ \\
\hline Event hold down & 5 & 5 & 5 \\
\hline Clear hold down & CPP $>60 ; 5 \mathrm{~m}$ & BPs $>90 ; 10 \mathrm{~m}$ & BPs $>90 ; 15 \mathrm{~m}$ \\
\hline
\end{tabular}

Kaunas, Events $=73$, Patients $=26$

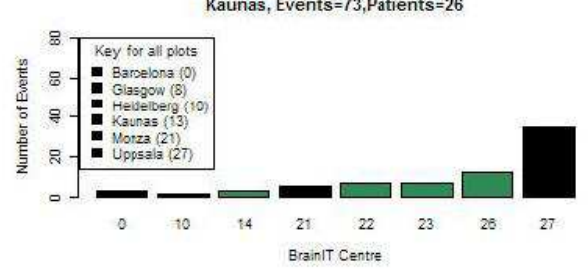

Monza, Events $=168$, Patients $=59$

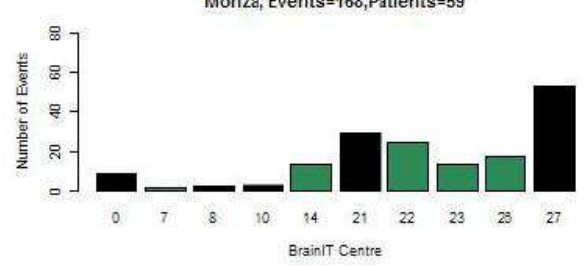

Uppsala, Events $=1166$, Patients $=100$

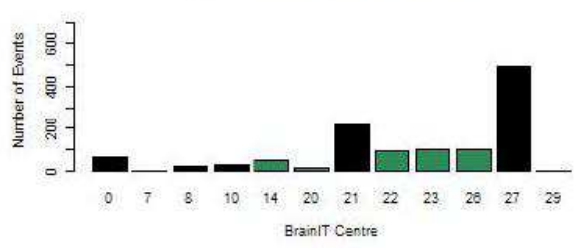

Barcelona, Events $=149$, Patients $=59$

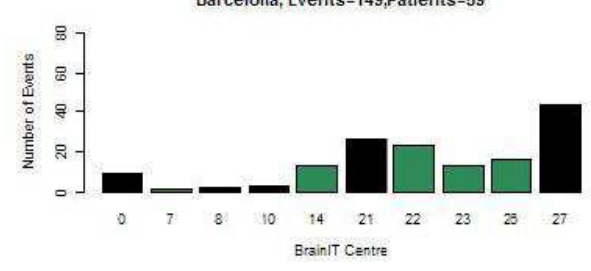

Heidelberg, Events $=607$,Patients $=81$

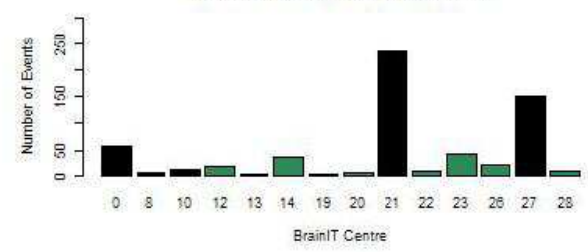

Glasgow, Events $=1770$,Patients $=111$

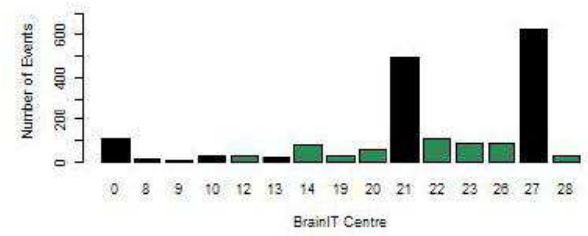

Figure 2. Number of events by BrainIT centre

Table 2. Event definitions for further analysis

\begin{tabular}{|c|c|c|}
\hline Parameters & BPs Event Holddown & BPs Clear Holddown \\
\hline BPs $<90$ OR BPm $<70$ & 5 & 5 \\
BPs $<100$ OR BPm $<70$ & 5 & 5 \\
BPs $<90$ OR BPm $<70$ & 2 & 2 \\
BPs $<100$ OR BPm $<70$ & 2 & 2 \\
\hline
\end{tabular}

continue using parameters from a published study that can be justified to colleagues in the medical community. 
Table 3. Event classification ( $T=$ Trigger, $C=$ Clear $)$

\begin{tabular}{|c|c|}
\hline BPs & BPm \\
\hline T-BPs-C-BPs-BPm-Enclosed (1) & T-BPm-C-BPm-BPs-Enclosed (5) \\
T-BPs-C-BPs-BPs-Only (2) & T-BPm-C-BPm-BPm-Only (6) \\
T-BPs-C-BPm (3) & T-BPm-C-BPs (7) \\
T-BPs-C-Both (4) & T-BPm-C-Both (8) \\
\hline \multicolumn{2}{|c|}{ Both } \\
\hline T-Both-C-BPs-Enclosed (9) \\
T-Both-C-BPm-BPs-Enclosed (10) \\
T-Both-C-Both (11)
\end{tabular}

(b) Identifying Causes of Adverse Events

The parameters studied so far to produce the hypotension event results above, have been the following measures of blood pressure:

- Systolic (BPS) the maximum pressure when the heart contracts and blood begins to flow.

- Diastolic (BPD) the minimum pressure, occurring between heartbeats.

- Mean (BPM) a combination of the two above, most often calculated as $B P D+(B P S-B P D) / 3$.

There are eleven different profiles that characterise the detection of events in the study, which are summarised in table 3. An important result to date has been the large number of Type- 6 events occuring within this representative data-set - where an event is triggered and held down by BPm only (see figure 3 ). The implication from this result is that many hypotensive events are being missed by centres that only measure BPs - a common practice, given that this issue of which pressure to measure is still subject to great debate in the clinical community.
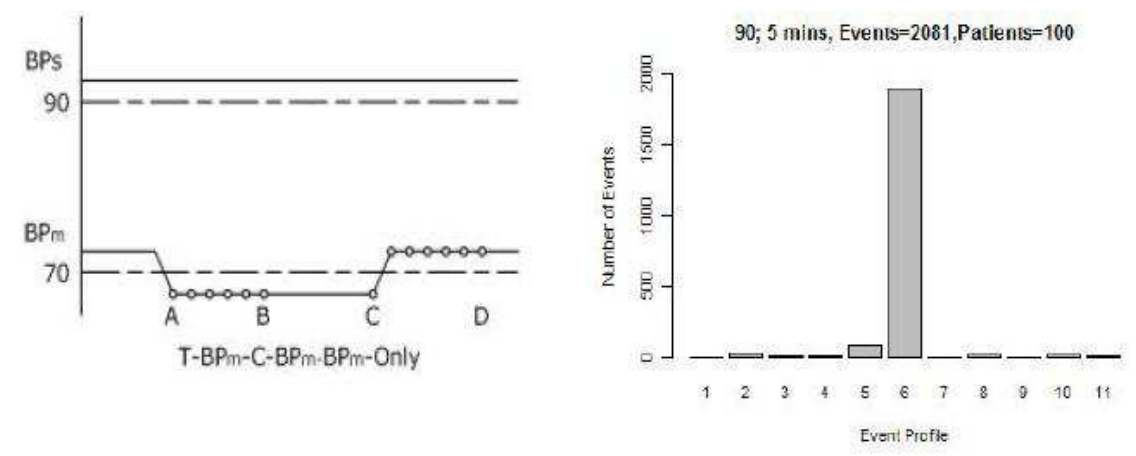

Figure 3. Type 6 event definition and number

The suggestion that the mean blood pressure should be given more weight than previously thought is an important research finding that will be investigated further throughout the project. 


\section{Computational Requirements}

There are three key steps to the delivery of the final system proposed in the AvertIT project:

1. The collection of data from the various distributed sources around Europe, contributing to the project.

2. The construction of a system that learns to recognise significant patterns in the variable indicator fields and associate them (correctly) with the onset of hypotension.

3. The implementation of an interface that will trigger an alarm based on the prediction of this event, and provide causative information to aid with the administration of treatment.

Steps 1 and 2 have fundamental research challenges, which must be addressed for the project to be successfully completed.

\section{(a) Data Federation}

Figure 4 shows the proposed architecture for the infrastructure to support the Avert-IT project.

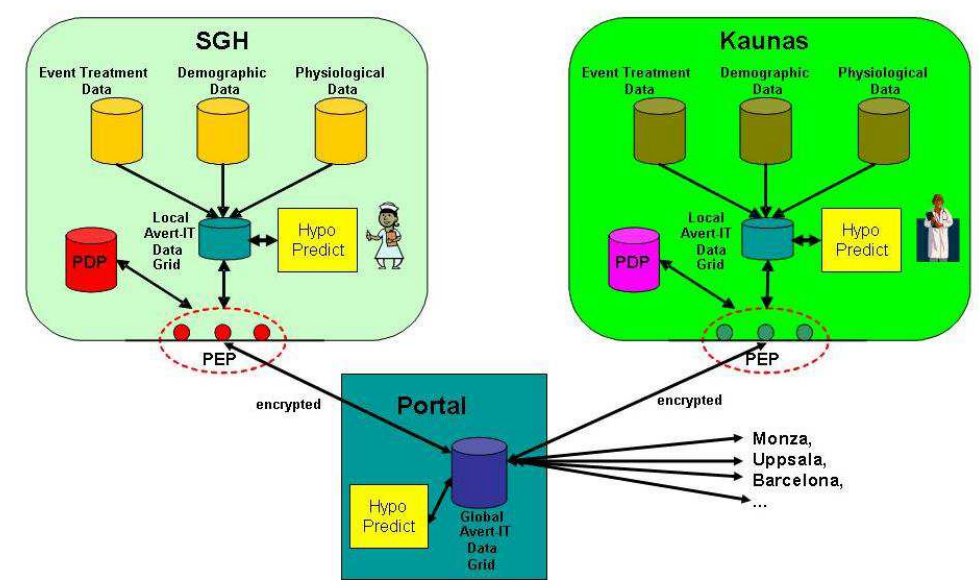

Figure 4. Avert-IT proposed infrastructure

The connection between non-clinical centres - the data grid provider and the front-end/ prediction-engine developer (grouped together in the blue box) - is relatively well-defined and can be achieved using either web services or direct JDBC calls, and once established to both parties' satisfaction can remain relatively static for the duration of the project.

However the connection to clinical systems must satisfy the constraints of a variety of health-centre policies, in terms of access to their data, secure transit of that data into the Avert-IT database, then the security of that data once it has left 
the perimeters of the centres involved. The most efficient method of establishing such connections, is to have a lightweight client - validated to the satisfaction of the centres involved - sitting within the firewall (or a de-militarized zone) of the centre, and which pushes data out to a receiving program run by the grid provider, which can then parse and upload to the Avert-IT database.

The first challenge in setting up a data grid over several heterogeneous clinical sources is to ascertain the structure of the data concerned. This takes the format of the data schema used, and also in this case, the nature of the data files themselves (often in the medical field, it is more than simply a case of connecting to a database), which include in this project ASCII text files, semi-structured XML and clinicalspecific Health-Level 7 format (HL7).

As the initial prototype system is developed, the volume of data is relatively low and, as is the nature of prototyping, any development issues can be overcome on a small-scale in the first instance. However when the scale of development increases and a production system is required to support the project, two major issues become evident: the method of securely connecting sources in real-time, and processing the large amount of data in transit over a feasible timescale.

\section{(b) Decision-Support for Event Prediction}

To accurately predict events, a decision-support module must be created, which takes account of the data retrieved so far, then makes a decision whether to send an alarm or not. There are a variety of methods of implementing a decision-support system, in addition to simple look-up tables and case-based reasoning, and these are listed below:

- Genetic Algorithms (GA) this is a search technique that involves generating a random selection of solutions across the domain, then testing this against a fitness function. The fittest solutions are then carried over to the next generation of solutions until a pre-determined level of generations has been completed. Though useful in scheduling and timetabling optimizations, there is some doubt as to whether the GA would be applicable in this system.

- Bayesian Belief Networks (BBN) this is a probabilistic model that represents a set of variables and their probabilistic dependencies. Inherently capturing probability in its development, training a BBN architecture with data-driven mechanisms has proved difficult.

- Artificial Neural Networks (ANN) this is a computer-simulated model of the brain, where individual neurons are interconnected from an input layer, through one or more hidden layers, to an output layer.

Balancing the project requirements and the various advantages and disadvantages of the different approaches, it has been agreed by the project consortium that a Bayesian approach to training an Artificial Neural Network (a BANN) would be the most effective way of training the system to detect the onset of a hypotensive event based on the input data of associated parameters. The motivation for considering the BANN is its application to the classification and modelling of highly nonlinear relationships whilst also considering probabilistic factors, expected to be 
a major aspect in the clinical inputs involved. Though the investigation of this area through comparison of the different methods would be of great benefit, given the limited time-scale of the project it was decided to choose one method most likely to yield useful results.

\section{Avert-IT Security}

The highly sensitive nature of the information being transferred and analysed in this project means that security is a paramount concern. Without the guaranteed security of the system, the likelihood of any widespread adoption will be remote. This section discusses the main issues that must be addressed to make this a secure and hence viable enterprise.

\section{(a) Patient Anonymity and Consent}

Most of the information required for the Avert-IT project does not require the identity of the patient to be known. In the first instance, all that is required are the health indicator measurements, the resultant outcome and the threshold information by which the relevant centre determines what constitutes a hypotensive event. Certain statistical demographic information is also required but nothing that can immediately identify patients.

However, the ethics of the project dictate that if a patient's data is to be used then they must give consent to that use. As such, a feedback loop must exist that allows the researcher to identify what patient data would be most useful to the study, requesting use of this data back to the providing centre, then obtaining consent for its use. In studies involving patients with traumatic brain injury, there is the added difficulty that subjects are incapacitated and cannot consent themselves. Instead Assent being obtained from relatives.

The data used in the first stage of this project are sets of example dummy data that cannot be linked to real patients. In the production stage, it is envisaged that any data leaving the bounds of the clinical centres will only have an identifier that only has meaning to the centre involved (a local ID).

\section{(b) Role-Based Access Control}

The delineation of duties and an agreement that outlines what recourse can be taken in place of a security breach is paramount. A necessary part of this agreement is to establish a policy of role-based access control that limits what parties can see what data, based on their role privilege.

In Avert-IT there is likely to be a coarse-grained hierarchy of roles, based on location within the project itself. The clinical centres should be able to see their own data, but not that of the other partners. The users at the end of the development process should also have a sub-set hierarchy based on their role within the environment using the system.

As the mediating agent bringing the data together, a pertinent question is whether NeSC, the data grid provider, should be able to view the data. This is a question that is yet to be resolved, but for which the VANGUARD solution (Virtual ANonymisation Grid for Unified Access to Remote Data) exists [18]. The 
VANGUARD systems allows data to be joined from two centres and presented to a final user, without having access to the data itself (through the exchange of cryptographic keys between end-user and data-provider).

\section{Implementation}

This section on implementation will provide the details in setting up the local unified grid infrastructure, which brings together the distributed data and makes it accessible to the development of the user interface and the Hypo-Predict engine.

\section{(a) Clinical End-Points}

There are six clinical centres involved in the Avert-IT project, each with idiosyncratic methods of recording and outputting patient data relating to hypotensive episodes. In addition, the comprehensive Brain-IT database is available for use by the Avert-IT project. These are the primary sources with which NeSC must interact in order to set up the unified data grid.

The results so far from study of the various systems architectures are as follows (the sources at Uppsala and Kaunas have yet to be analysed):

- Philips DocVu [19]: data is output from the ICU monitors and written to ASCII text files stored on a single server (per hospital) situated behind the firewall of the UK health service (NHS). Optional DocVu interfaces to the local hospital information system also provide basic patient demographic data and laboratory test results.

- CMA ICU-Pilot [20]: generally stored in proprietary format behind the campus firewall, there is an option to export the information to standard XML.

- Drager Medical [21]: one of the more sophisticated technologies, the output is sent to HL7 files, a standard adopted by the health community at large.

- BrainIT: this is a straight-forward Access database, hosted on the BrainIT secure area.

As can be seen the general format of the various systems is similar in that the same parameters are drawn, but they are stored in very different schemas. Therefore, the data in its raw format must be translated into a standard description.

\section{(b) Avert-IT Data Grid}

The output of the data grid takes the form of a simple database that stores selected patient demographic information, centre details (in effect an audit trail of the data history) and the parameters themselves. The required core parameter values as identified in the BrainIT database (which has been published as the predominant standard in the field [22]) are: Heart rate (HRT), Respiration rate (RR), Mean blood pressure (BPM), Systolic blood pressure (BPS), Diastolic blood pressure (BPD), Blood oxygen concentration (SAO2), Temperature (TC), Mean intra-cranial pressure (ICPM), Cerebral perfusion pressure (CPP). 
As the project progresses and the investigation of contextual information develop, other parameters previously unused may become significant (e.g. End-tidal carbon dioxide - EtCO2). As such, the ability to modify this schema and its data is a requirement.

The processes involved in the parsing of the raw data files do still require separation when the system gets to production stage (and during the test phases). It is envisaged that the parsing described above will take place through a lightweight client application distributed to each centre, validated by their system administrators and held on their side of their own firewall. The parsing will then package the relevant data into WS-friendly (Web Services) XML ready for communication to NeSC, which will be analysed and uploaded.

The overall automation of this process is a non-trivial task and must take account of three key issues: identifying relevant patient information at the clinical centre, coping with the volume of data to be transported, and maintaining security at all stages in the process.

\section{(c) Progress to date and next steps}

The progress of the work, as of the date of submission (1st September 2008), is that example file outputs from four sources (the BrainIT database, Glasgow, Heidelberg and Monza clinical data systems) have been parsed and input into the grid database. This comprises a minimal example of the data grid, from which the front-end and BANN developer can draw parameters from. The JDBC connection between these two parties has been established and functions correctly. The interface to the data grid can be viewed through a portal at http://avertit.nesc.gla.ac.uk:18080/gridsphere.

The next steps have been identified as sourcing sample files from the other clinical partners (Barcelona, Uppsala and Kaunas) and following a similar pattern in uploading the relevant data parameters.

Between the clinical sources and the data grid provider, the parsing code that currently exists will need to be decomposed into packaging of Web Service XML files and negotiations will need to proceed into allowing this communication between dedicated servers across firewalls. It is likely that business cases will need to be compiled to defend the need for this cross-institution communication, and the focus of such cases will of course be on the security of the system.

\section{Conclusions}

The Avert-IT project proposes the development of a system architecture that can automatically predict adverse hypotensive events over a useful timescale, without the intervention of a health-care professional.

The results of clinical research performed over the BrainIT database in conjunction with various definitions of hypotensive event thresholds have suggested that a value of $90 \mathrm{mmHg}$ over 5 mins (one of the EUSIG definition) is the optimal hypotension threshold definition to proceed with, and that positive research results have been obtained, which provide evidence of the desirability of uniformly measuring mean and systolic blood pressure together - a practice still subject to debate in the clinical community.

Article submitted to Royal Society 
The computational requirements, along with the implementation so far, of setting up a grid infrastructure and passing this data to a decision-support tool have been discussed, along with the different types of support tools available, the BANN being preferred, due to its probabilistic abilities. The major security issues in unifying and using such data have been discussed, with the identification of static agreements and role-based policies being requirements before the Avert-IT system gets anywhere near production level.

Research to date has already produced useful information in the investigation of the causes and definitions of hypotension in patients. It is hoped that with the successful completion of the infrastructure outlined here that the Avert-IT project will produce a highly innovative system that will have a great impact on the field of clinical health-care.

\section{Acknowledgements}

The authors would like to acknowledge the EU FP7 program (grant number 217049), kindly funding the Avert-IT project.

We would also like to acknowledge the work of the BrainIT group investigators and participating centres to the BrainIT dataset without whom this work could not have been conducted:

Barcelona, Spain: Prof Sahuquillo; Cambridge, UK: Prof. Pickard; Edinburgh, UK: Prof. Whittle; Glasgow, UK: Mr. Dunn; Gothenburg, Sweden: Dr. Rydenhag; Heidelberg, Germany: Dr. Kiening; Iasi, Romania: Dr. Iencean; Kaunas, Lithuania: Prof. Pavalkis; Leipzig, Germany: Prof. Meixensberger; Leuven, Belgium: Prof. Goffin; Mannheim, Germany: Prof. Vajkoczy; Milano, Italy: Prof. Stocchetti; Monza, Italy: Dr. Citerio; Newcastle upon Tyne, UK: Dr. Chambers; Novara, Italy: Prof. Della Corte; Southampton, UK: Dr. Hell; Uppsala, Sweden: Prof. Enblad; Torino, Italy: Dr. Mascia; Vilnius, Lithuania: Prof. Jarzemaskas; Zrich, Switzerland: Prof. Stocker.

\section{References}

Avert-IT project proposal Advanced Arterial Hypotension Adverse Event prediction through a Novel Bayesian Neural Network (available at http://wiki.avertit.org/wordpress)

Odin Browser Program T.P. Howells, I.R. Piper, P.A. Jones, M. Souter, and J.D. Miller. Design of a research database for the study of secondary insults following head injury. Journal of Neurotrauma, 12:471-472, 1995.

Tarassenko et al., BioSign: multi-parameter monitoring for early warning of patient deterioration. $3^{\text {rd }}$ IEE International Seminar on Medical Applications of Signal Processing (2005/11199), p71-76

Philips Medical Event Surveillance Monitor: http://www.medical.philips.com/main/ products/patient-monitoring/assets/docs/event-surv4522-982-91371.pdf

Geoffrey Manley, M. Margaret Knudson, Diane Morabito, Susan Damron, Vanessa Erickson, and Lawrence Pitts. Hypotension, hypoxia, and head injury. ARCH SURG, 136:11181123, 2001.

A. Marmarou, R.L. Anderson, J.D. Ward, et al. Impact of ICP instability and hypotension on outcome in patients with severe head trauma. Journal of Neurosurgery, 75:S59S66, 1991. 
European Society of Cardiology, Guidelines on the management of hypertension. European heart journal 2007, 28; 1462

Chesnut RM, Marshall LF, Klauber MR, et al. The role of secondary brain injury in determining outcome from severe head injury. J Trauma 1993;34:216-222

J. Piek, Guidelines for the intensive care management of patients with Head Injury Intensive care medicine 1998, 28; 1221

Stochetti N, Penny K, Dearden M, Braakman R, Cohaon F, Iannotti F, Lapierre F, Karimi A, Maas A, Murray G, et al. Intensive care management of head-injured patients in Europe: a survey from the European Brain Injury Consortium. Intensive care med (2001) 27: 400-406

Bulger E, Nathens A, Avery B, Rivara F, Moore M, Mackenzie E, Jurkovich G. Management of severe head injury: institutional variations in care and effect on outcome. Critical Care Med 2002; 30: 1870-1876

BrainIT: http://www.brainit.org

R.O. Sinnott, I. Piper, e-Infrastructures Fostering Multi-Centre Collaborative Research into the Intensive Care Management of Patients with Brain Injury, Handbook on Research on Information Technology, November 2008.

Donald R, Event Definition Analysis Preliminary Results, Avert-IT progress report, February 2008 (available at http://avertit.wordpress.com)

Patricia Jones, Peter Andrews, Susan Midgley, Shirley Anderson, Ian Piper, Janis Tocher, Alma Housley, Jane Corrie, James Slattery, Mark Dearden, and Douglas Miller. Measuring the burden of secondary insults in head-injured patients during intensive care. Journal of Neurosurgical Anesthesiology, 6:4-14, 1994.

$[15]$

Donald R, Avert-IT BANN Associated Parameter Selection, Avert-IT progress report, June 2008 (available at http://avertit.wordpress.com).

Robert H Dolin, et al. - HL7 Clinical Document Architecture. Journal of American Medical Informatics Association, 2001;8:552-569

Sinnott R, Ajayi O, Stell A, and Young A: Towards a Virtual Anonymisation Grid for Unified Access to Remote Clinical Data, Proceedings for HealthGrid 2008, Chicago. [18]

DocVu: $\quad$ http://www.medical.philips.com/main/products/patientmonitoring/products/doc-center/

CMA Microdialysis ICU-Pilot http://www.icupilot.com

Drager Medical http://www.draeger.com

Piper I, Citerio C, Chambers I et al. The BrainIT Group: Concept and Core Dataset Definition. Acta Neurochir 145:615-629 2003.

Donald R, Event Definition Analysis - Using EUSIG Hypotension definitions, Avert-IT progress report, May 2008 (available at http://avertit.wordpress.com)

\section{List of Figure Captions}

1. Hypotensive event definition

2. Number of events by BrainIT centre

3. Type-6 event definition and number

4. Avert-IT proposed infrastructure 


\section{University Library}

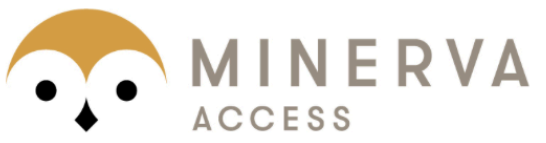

\section{A gateway to Melbourne's research publications}

Minerva Access is the Institutional Repository of The University of Melbourne

\section{Author/s:}

STELL, ANTHONY; SINNOTT, RICHARD; Jiang, Jipu; Donald, Rob; Chambers, Iain; Citerio, Giuseppe; Enblad, Per; Gregson, Barbara; Howells, Tim; Kiening, Karl; Nilsson, Pelle;

Ragauskas, Arminas; Sahuquillo, Juan; Piper, Ian

Title:

Federating distributed clinical data for the prediction of adverse hypotensive events

Date:

2009

\section{Citation:}

Stell, A., Sinnott, R., Jiang, J., Donald, R., Chambers, I., Citerio, G., et al. (2009). Federating distributed clinical data for the prediction of adverse hypotensive events. Royal Society of London Philosophical Transactions A: Mathematical, Physical and Engineering Sciences, 367 (1898), 2679-2690.

\section{Publication Status:}

Published

\section{Persistent Link:}

http://hdl.handle.net/11343/28772

\section{File Description:}

Federating distributed clinical data for the prediction of adverse hypotensive events 


\section{University Library}

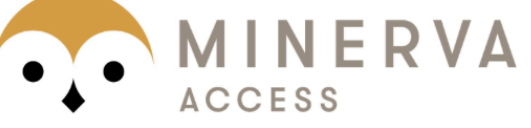

A gateway to Melbourne's research publications

Minerva Access is the Institutional Repository of The University of Melbourne

\section{Author/s:}

Stell, A;Sinnott, R;Jiang, J;Donald, R;Chambers, I;Citerio, G;Enblad, P;Gregson, B;Howells, T;Kiening, K;Nilsson, P;Ragauskas, A;Sahuquillo, J;Piper, I

Title:

Federating distributed clinical data for the prediction of adverse hypotensive events

Date:

2009

\section{Citation:}

Stell, A., Sinnott, R., Jiang, J., Donald, R., Chambers, I., Citerio, G., Enblad, P., Gregson, B., Howells, T., Kiening, K., Nilsson, P., Ragauskas, A., Sahuquillo, J. \& Piper, I. (2009). Federating distributed clinical data for the prediction of adverse hypotensive events.

Philosophical Transactions of the Royal Society A: Mathematical, Physical and Engineering Sciences, 367 (1901), pp.2679-2690. https://doi.org/10.1098/rsta.2009.0138.

Persistent Link:

http://hdl.handle.net/11343/30028 